\title{
DYNAMIQUE DES MATIERES EN SUSPENSION DANS UN BIEF DE LA RIVIERE LOT
}

\author{
par J.-C. Massio (1) et H. Décamps (1)
}

\section{RESUME}

Des bilans amont-aval ont été réalisés sur le bief de Cajarc: réservoir artificiel de $3 \mathrm{~km}$ de long et $1269000 \mathrm{~m}^{3}$ sur la rivière Lot. Les paramètres suivants ont été étudiés sur des cycles de 24 heures pour différents débits : matières minérales et organiques en suspension, turbidité de l'eau, teneurs en pigments chlorophylliens, en nitrates, nitrites et phosphates.

Pour des débits inférieurs à $80 \mathrm{~m}^{3} / \mathrm{s}$, on observe une tendance à la décantation des matières minérales en suspension, et pour des débits supérieurs à $100 \mathrm{~m}^{3} / \mathrm{s}$ une tendance à l'arrachement des sédiments. Ces mécanismes varient selon la saison.

La majorité des matières organiques en suspension est constituée en hiver de détritus issus du bassin versant et du lit de la rivière. En été, la part des algues devient importante mais demeure faible relativement aux teneurs observées plus en aval dans la rivière.

Dans les bilans amont-aval, l'évolution des teneurs en matières organiques et pigments chlorophylliens d'une part et des teneurs en nitrates, phosphates et nitrites d'autre part rend compte des phénomènes de biosynthèse et de biodégradation au sein de la masse d'eau.

(1) Laboratoire d"Hydrobiologie, Université $P$. Sabatier, 118 route de Narbonne, 31077 Toulouse Cedex. 
En novembre $\left(155 \mathrm{~m}^{3} / \mathrm{s}\right)$ et en septembre $\left(46 \mathrm{~m}^{3} / \mathrm{s}\right)$, la biodégradation est prépondérante. En février $\left(166 \mathrm{~m}^{3} / \mathrm{s}\right)$, on observe, après une biodégradation dominante, un équilibre en fin de journée. En mai $\left(55 \mathrm{~m}^{3} / \mathrm{s}\right)$, la biosynthèse domine pendant le jour. II en est de même en juin $\left(65 \mathrm{~m}^{3} / \mathrm{s}\right)$ tant que la turbidité n'est pas trop importante.

\section{INTRODUCTION}

L'ensemble des matières minérales et organiques transporté dans une rivière évolue en quantité et en qualité de l'amont vers l'aval. II a été possible en de nombreux cas d'étudier le matériel transporté par un fleuve ou une grande rivière (PETROVIC 1969, GOLTERMAN 1973, BRANSKI 1974) ou encore la capacité de piègeage de tel ou tel réservoir, le plus souvent de grandes dimensions (ROEHL et HOLEMAN 1973).

Peu d'études concernent de petits réservoirs, assimilables à des biefs avec un faible temps de rétention comme on en trouve fréquemment sur le parcours des rivières. En outre, les bilans sont gènéralement réalisés sur de langues périodes de temps, dans le but d'évaluer globalement les transports et non les interactions entre les différents types d'éléments transportès.

Les mesures amont-aval réalisées au cours de cette étude étaient destinées à suivre la dynamique des matières en suspension dans un réservoir de faible capacité, sur des courtes périodes de temps, et à différents mois de l'année.

\section{LE BIEF DE CAJARC}

Le bief étudié forme un petit réservoir dans la partie moyenne du Lot canalisé (BORDES 1973, DAUTA 1975). II s'étend sur $2500 \mathrm{~m}$ de longueur entre une chaussée submergée et un barrage aval. Ses caractéristiques sont les suivantes:

- aire : $357000 \mathrm{~m}^{2}$,

- capacité : $1269000 \mathrm{~m}^{3}$.

- profondeur maximale: $7,4 \mathrm{~m}$.

- largeur moyenne: $95 \mathrm{~m}$,

- bassin drainé : $7350 \mathrm{~km}^{2}$ (soit $62 \%$ du bassin de la rivière Lot).

Le plan d'eau ainsi formé est utilisé pour diverses activités de loisir (le nautisme notamment).

Le barrage est à vannes relevables. L'usine fonctionne au fil de l'eau, c'est-à-dire à niveau constant et peut turbiner jusqu'à $165 \mathrm{~m}^{3} / \mathrm{s}$. Au-delà l'eau passe en majorité sous le barrage dont les vannes sont relevées au fur et à mesure de l'augmentation du débit.

\section{METHODES}

Etant donné la présence de zones d'eau morte assez étendues, le volume d'eau renouvelable a été ramené à $941000 \mathrm{~m}^{3}$ (la capacité étant de $1269000 \mathrm{~m}^{3}$ ). 
Les temps théoriques de rétention ont été calculés à partir de ce volume et des débits horaires fournis par l'usine installée sur le barrage.

Après filtration, les données brutes = perte au feu " et a résidu non volatil * ont été assimilées respectivement à la matière organique et à la matière minérale. Une correction a donc été nécessaire pour tenir compte de la teneur en cendres (entre 10 et $15 \%$ ).

La teneur en pigments chlorophylliens a été évaluée après centrifugation, par spectrophotométrie. La turbidité a été évaluée au moyen d'un turbidimètre Hach. L'azote des nitrates $\mathrm{N}-\mathrm{NO}_{3}$ a été dosé par spectrophotométrie en utilisant du Nitraver IV. L'azote des nitrites $\left(\mathrm{N}-\mathrm{NO}_{2}\right)$ a été obtenu par dosage au spectrophotomètre de la coloration obtenue en ajoutant de l' $\alpha$-naphtylamine au composé diazoté formé par les nitrites et l'acide sulfanilique. Le phosphore des phosphates ( $\left.\mathrm{P}-\mathrm{PO}_{4}\right)$ a été obtenu par dosage au spectrophotomètre après réduction au chlorure stanneux du complexe phosphomolybdique extrait par l'isobutanol.

\section{RESULTATS}

Les résultats sont regroupés dans les figures 1 à 5 . Pour chacun des paramètres considérés, les tracés en traits épais représentent les valeurs amont, et ceux en traits fins les valeurs aval. Ces dernières ont été décalées de façon à ce que figurent sur une même verticale les valeurs concernant théoriquement la même eau, en regard de l'horaire de passage au point amont. L'aire entre les tracés amont et aval a été hachurée lorsque la valeur aval était inférieure à la valeur amont.

Les 22-23 septembre 1974, pour un débit moyen de $46 \mathrm{~m}^{3} / \mathrm{s}$, on observe une diminution des teneurs en matières minérales $(\mathrm{MM})$ et matières organiques (MO) au cours de la traversée du bief. De même, les teneurs en pigments chlorophylliens diminuent pendant l'après-midi et la nuit pour augmenter le matin en fin d'observation. Corrélativement, les nitrates et les phosphates augmentent pendant la première journée et la nuit: la biodégradation prédomine et ne cesse qu'au matin lorsque le bilan en pigments s'inverse. 


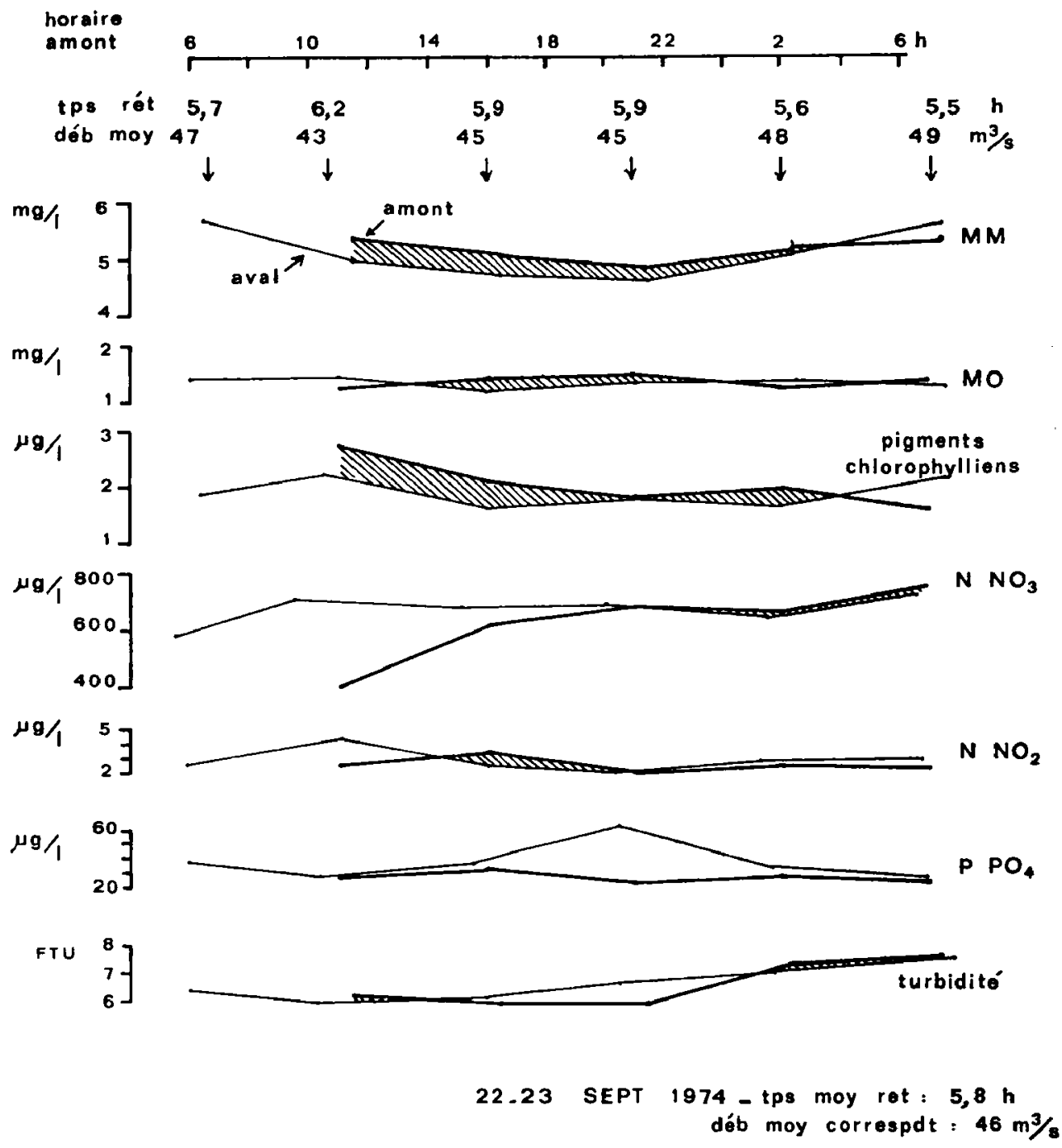

Figure 1 : Evolution des différents paramètres entre l'amont et l'aval le 22-23 septembre 1974. tps rét.: temps de rétention; déb. moy.: débit moyen: $M M$ : matières minérales; $\mathrm{MO}$ : matières organiques. Les zones hachurèes marquent la diminution entre l'amont et l'aval. 
Le 5-6 novembre 1974, lors d'une période de fort débit établi durant plusieurs semaines, l'eau se charge en matières minérales et organiques entre l'amont et l'aval, en même temps que la turbidité augmente. Les teneurs en pigments chlorophylliens, toujours trés faibles, diminuent entre l'amont et l'aval, à l'inverse de la matière organique. Cette dernière est constituée, dans sa plus grande partie, de matière remise en suspension à partir du sédiment. On observe dans l'ensemble, une augmentation des nitrates, à l'exception d'une mesure nocturne.

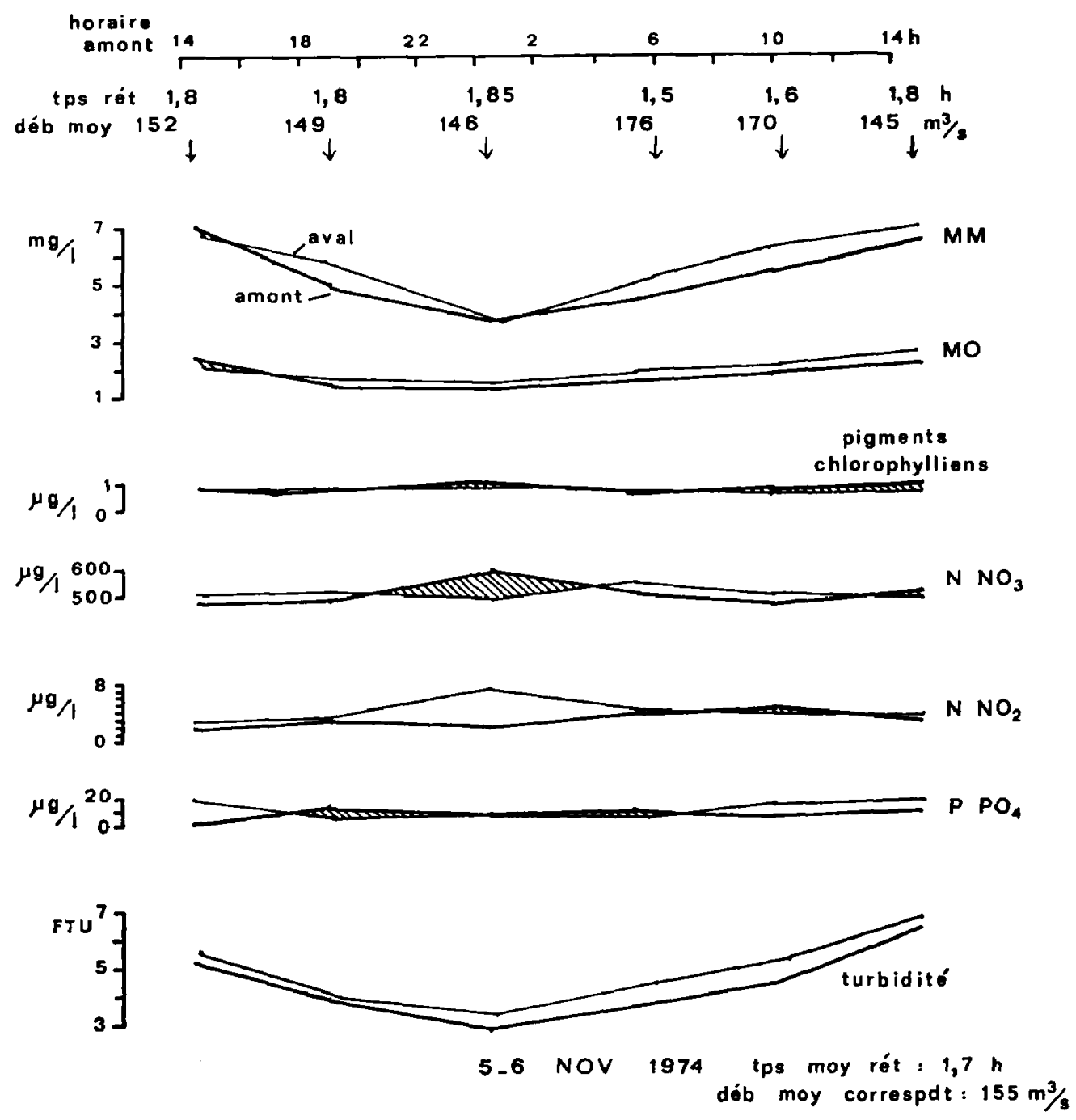

Figure 2 : Evolution des différents paramètres entre l'amont et l'aval le 5-6 novembre 1974

Le 18-19 février 1975, pour un débit semblable, les tendances sont identiques à celles de la période précédente. La diminution de la teneur en matières organiques dans la matinée du 19 est peut être due à une taille différente des 
particules. A la phase de biodégradation observée le 18, en fin de journée, fait suite une tendance à l'équilibre ainsi que le montrent les bilans en $\mathrm{NO}_{3}, \mathrm{PO}_{4}$ et $\mathrm{NO}_{2}$.

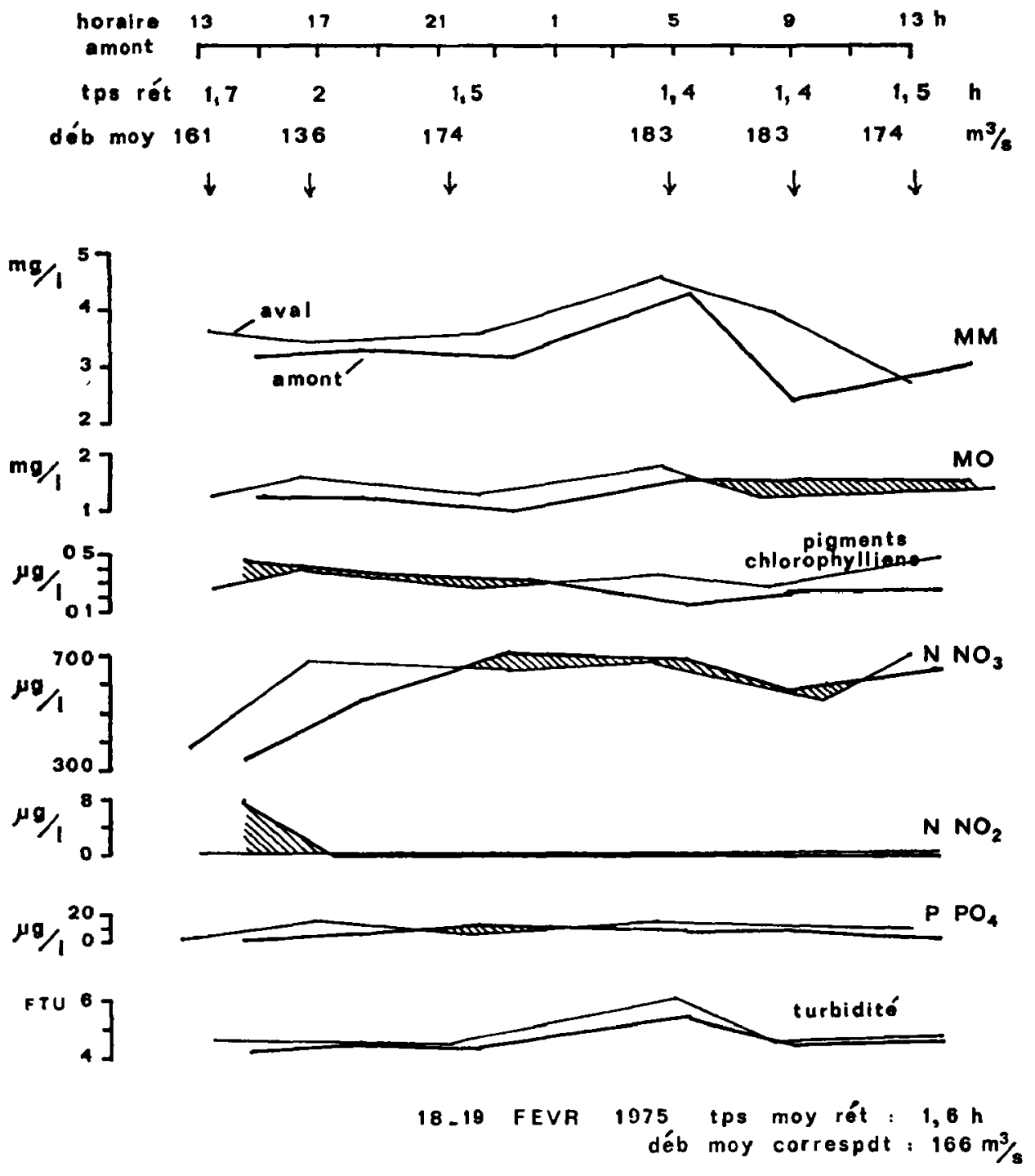

Figure 3 : Evolution des différents paramètres entre l'amont et l'aval le 1819 février 1975.

Le 20-21 mai 1975, le débit moyen est de $55 \mathrm{~m}^{3} / \mathrm{s}$. Les matières minérales et la turbidité diminuent au cours de la traversée du bief. L'évolution de la matière organique ressemble à celle des pigments chlorophylliens: les algues représentent la plus grande partie de la matière organique transportée. L'augmentation nocturne de matière organique et de pigments est sans doute due à des apports constants de zones d'eau morte, joints à une forte activité bactérienne. L'évolution des teneurs en nitrates traduit une prépondérance de la biosynthèse pendant 
la journée et une partie de la nuit, suivie d'une phase de biodégradation en fin de nuit. Toutefois, les variations de $\mathrm{N}-\mathrm{NO}_{2}$ et $\mathrm{P}-\mathrm{PO}_{4}$ montrent que la biodégradation demeure importante pendant la journée.
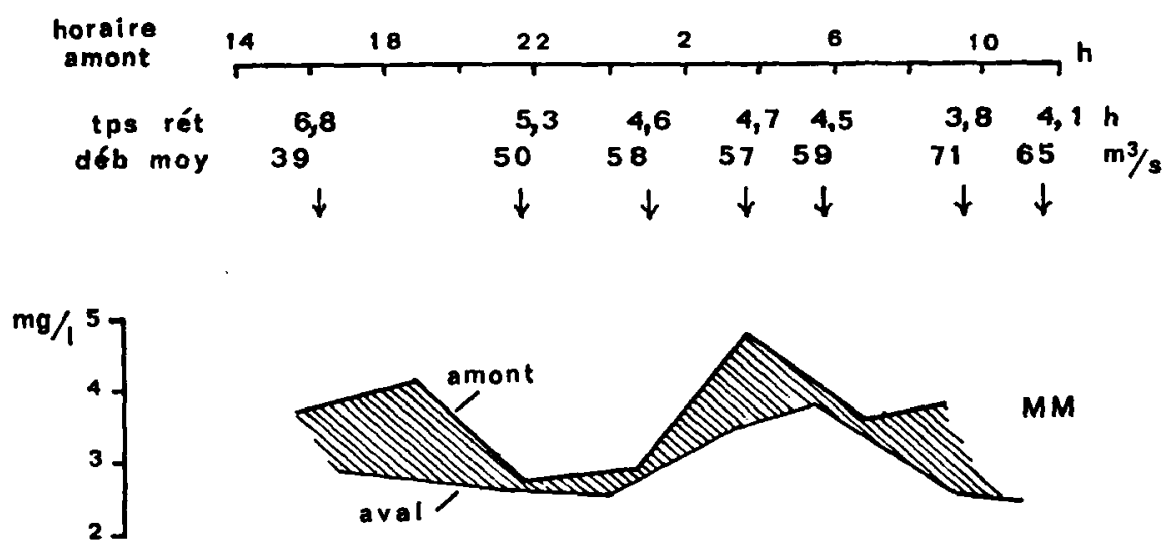

$\mathrm{mg} / \mathrm{I}_{2}$
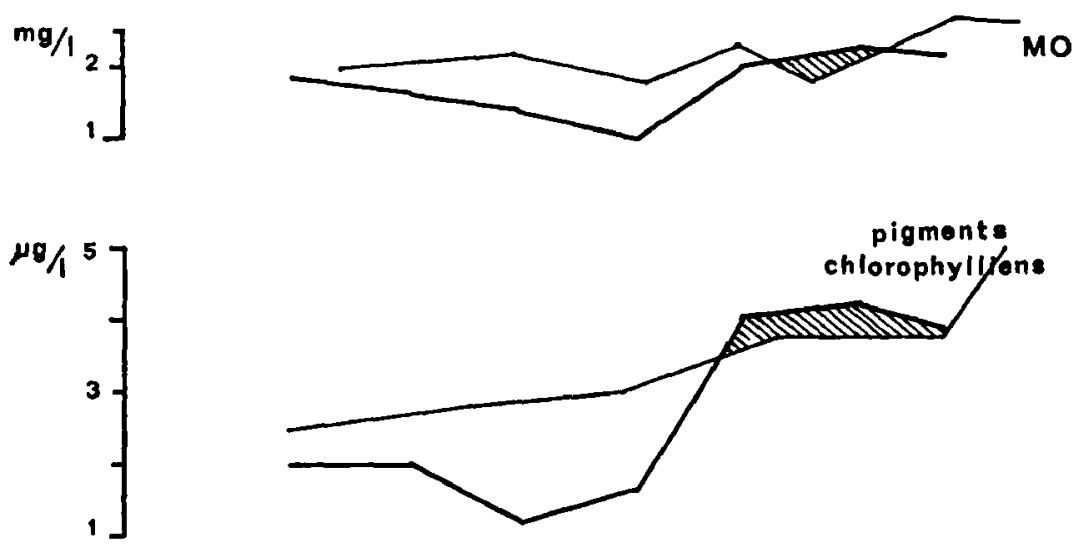

$$
\left.\mu g_{200}^{400}\right]
$$

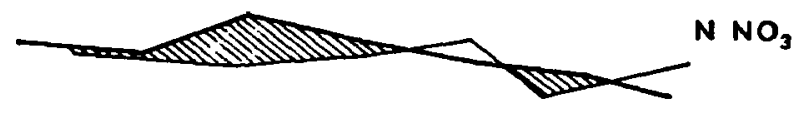

$$
\left.\begin{array}{r}
\mu g / 1 \\
{ }^{5} \\
2
\end{array}\right]
$$
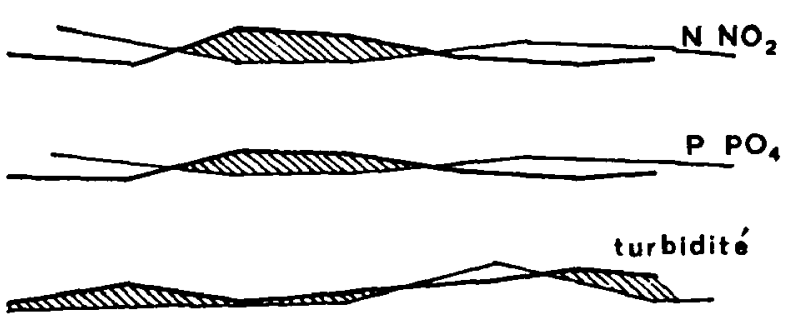

$$
\begin{array}{r}
\text { 20_21 MAI } 1975 \text { tps moy rét : } 4,8 \mathrm{~h} \\
\text { déb moy correspdt }=55 \mathrm{~m} / 3
\end{array}
$$

Figure 4: Evolution des différents paramètres entre l'amont et l'aval le 2021 mai 1975. 

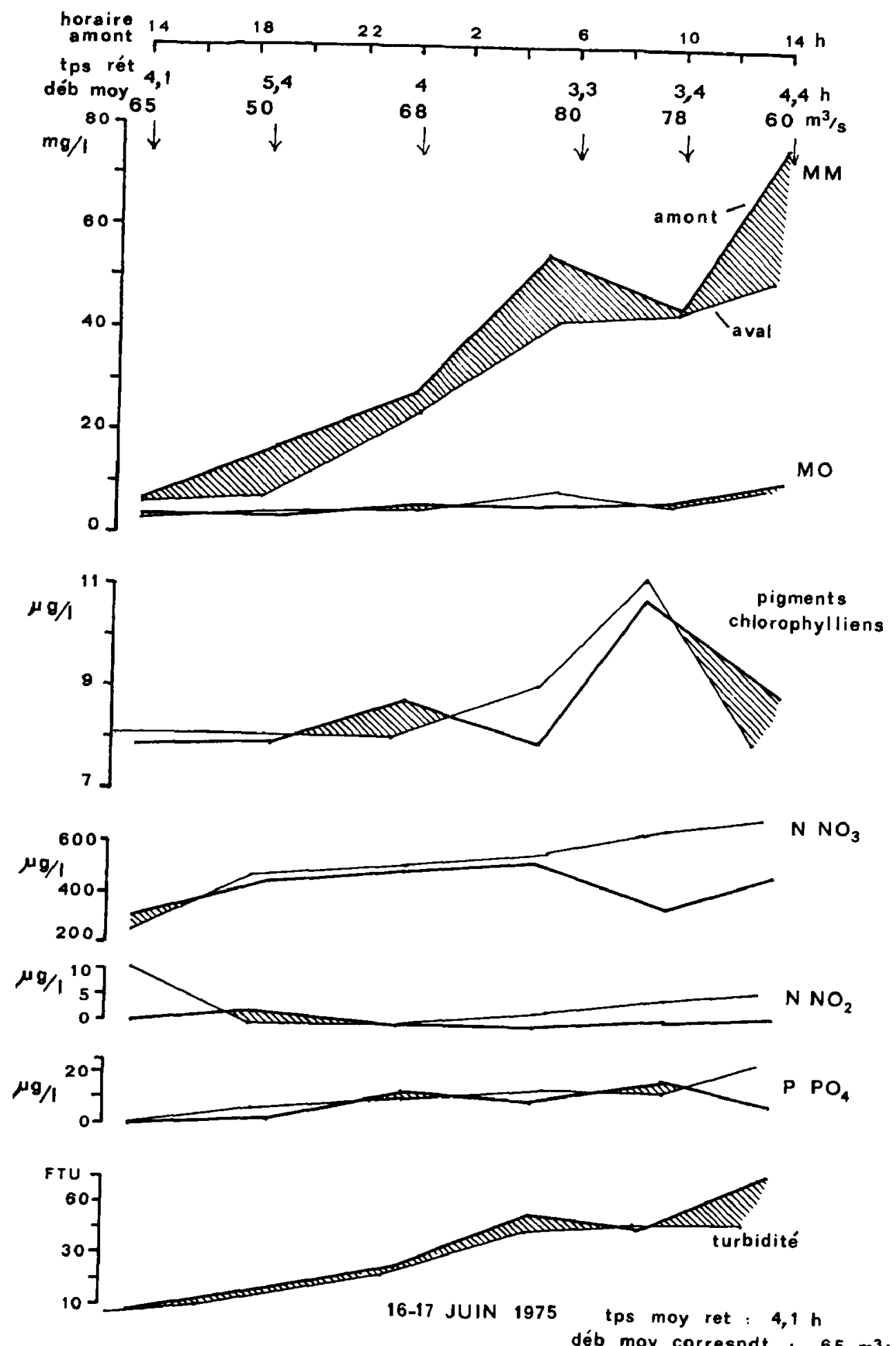

Figure 5: Evolution des différents paramètres entre l'amont et l'aval le 16 -
97 juin 1975 . 
Le 16-17 juin 1975, le débit moyen étant de $65 \mathrm{~m}^{3} / \mathrm{s}$, les eaux sont considérablement chargées en matières minérales à la suite de pluies sur une partie du haut bassin (vallée du Dourdou). Au cours de la période d'observation, l'eau arrivant à l'amont du bief est de plus en plus chargée en matières minérales. Une décantation importante se produit au cours de la traversée du bief: les dépôts peuvent être évalués à $500-5000 \mathrm{~kg} / \mathrm{ha}$. La turbidité augmente en conséquence. Les teneurs en matières organiques et pigments chlorophylliens évoluent de façon semblable. En fait, l'évolution de la biomasse algale dans le bief détermine le bilan global de la matière organique. Notons que ces algues de printemps (essentiellement des Diatomées) ne sont gênées dans leur développement que par les turbidités les plus élevées (environ $50 \mathrm{FTU}$ ). Au-dessous de ce seuil, elles se développent normalement dès le début du jour. Les teneurs en nitrates n'indiquent une biodégradation nette qu'en fin d'observation, pour les turbidités les plus élevées. Parallèlement, le pourcentage de phéophytine passe de 20 à $80 \%$.

La capacité de piégeage du réservoir a été évaluée en divisant la différence entre les teneurs en matières minérales à l'amont et à l'aval par la teneur en matières minérales à l'amont. Les pourcentages ainsi obtenus sont regroupés dans la figure 6 en fonction des débits moyens (et des vitesses moyennes correspondantes). A la tendance à la décantation observée pour les débits moyens les plus faibles s'oppose une tendance à l'enlèvement des matières minérales pour les débits moyens les plus forts. La valeur critique est comprise entre 80 et $100 \mathrm{~m}^{3} / \mathrm{s}$, c'est-à-dire aux environs d'une vitesse moyenne de $0,25 \mathrm{~m} / \mathrm{s}$. On doit toutefois noter l'existence de fortes variations du pourcentage de matières minèrales retenues ou relâchées pour un même débit.

\section{DISCUSSION}

Les matières en suspension dans l'eau et l'eau elle-même présentent des dynamiques distinctes. En juin, l'augmentation de matières minérales en suspension (et corrélativement de la turbidité) coincide avec des débits relativement stables. En février et mai, les variations de teneur en matières minérales ne peuvent pas être corrélées à des variations du débit.

En outre l'hétérogénéité de l'eau circulant dans le réservoir complique l'interprétation des mesures amont-aval. Par exemple, une légère instabilité du débit en fin de journée le 20 mai entraine dans l'écoulement des masses d'eau qui étaient localement isolées: ceci conduit à une augmentation nocturne des pigments chlorophylliens entre l'amont et l'aval.

En dépit de ces difficultés, dues à la nature même de l'écosystème rivière. plusieurs conclusions apparaissent.

Le poids de matières minérales en suspension, assez constant si l'on ne considere que les quatre premiers bilans, peut subitement augmenter comme en témoigne le bilan du mois de juin. Dans une rivière canalisée telle que le Lot, la charge en matières minérales ne dépend pas uniquement du débit : d'importants lâchages à partir des réservoirs situés en amont n'augmentent que faiblement la quantité de sédiments transportés au niveau de Cajarc. Par contre, des crues locales sur des affluents drainant des bassins où l'érosion est importante jouent un rôle prépondérant: les fortes teneurs en matières minérales des 16 et 17 juin sont consécutives à des pluies sur la vallèe du Dourdou, affluent du Lot. 
Le pourcentage de sédiment retenu dans le réservoir de Cajarc varie largement au cours d'une journée pour un même débit moyen. Même pour de faibles débits, les valeurs obtenues (fig. 6) sont très inférieures à celles de réservoirs comparables étudiés aux U.S.A., pour lesquels les pourcentages varient entre 33 et plus de $99 \%$ et sont le plus souvent supérieurs à $80 \%$ ROEHL et HOLEMAN 1973. HEINEMAN 1973). Les faibles temps de rétention existant lors des observations et le mode de fonctionnement du barrage sont probablement responsables de cette faible capacité de piégeage. L'éloignement des parties du bassin versant où les matériaux sont facilement érodés peut également expliquer cette différence.
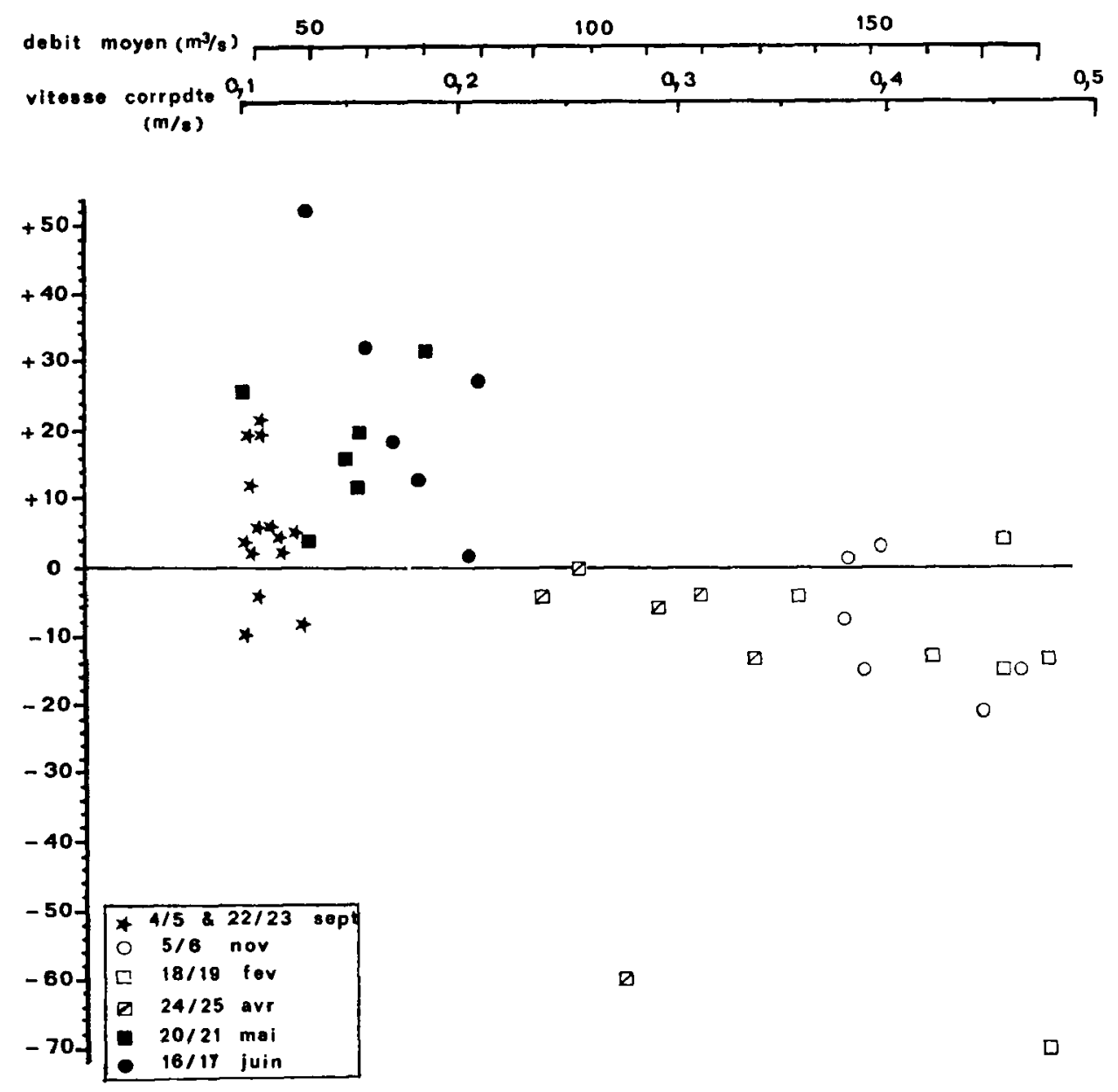

Figure 6 : Pourcentage de matière minérale retenue dans le bief $(>0)$ et enlevée du bief $(<0)$ aux différents débits considérés.

Pour des débits supérieurs à $80-100 \mathrm{~m}^{3} / \mathrm{s}$, l'eau s'enrichit en matières minérales lors de la traversée du bief de Cajarc: une partie du sédiment est remise en suspension mais de grandes variations peuvent, ici encore, être observées au cours de la journée. 
Les débits considérés dans cet article sont tous nettement supérieurs aux débits d'étiage. Dans ces conditions, la production primaire dans le réservoir est plus limitée par les faibles temps de rétention (entre 1,6 et 5,8 h) et par la turbidité (le 17 juin par exemple) que par les teneurs en nitrates et en phosphates.

Les périodes de biosynthèse se traduisent par une augmentation des pigments chlorophylliens accompagnée par une diminution des nitrates, des phosphates et des nitrites. Ces tendances s'inversent durant les périodes de biodégradation. Plusieurs exemples peuvent être tirés des bilans présentés ici, notamment lorsque la plus grande partie de la matière organique est composée d'algues. Toutefois, les deux phases - biosynthèse et biodégradation - ne sont pas toujours aussi bien caractérisées dans les figures 1 à 5 par suite : $\mathrm{NH}_{4} ;$

- de la possibilité qu'ont les algues d'assimiler de l'azote provenant de

- de l'existence d'une biosynthèse hétérotrophe importante ;

- de perturbations apportées par la remise en suspension de la matière organique lors des crues les plus fortes, ou par la présence d'une forte turbidité.

Enfin, en ce qui concerne la gamme des débits élevés considérée dans ce travail, le débit lui-même ne peut pas être corrélé à la teneur en matières minérales en suspension, ni par suite, à la turbidité. La seule variation globale observée est, dans l'ensemble, une diminution de la turbidité au-dessous du seuil de $80-100 \mathrm{~m}^{3} / \mathrm{s}$, et une augmentation au-dessus de ce seuil.

D'un point de vue méthodologique, il parait important de souligner que dans un réservoir de faible capacitè tel que celui de Cajarc, et dont le temps de rétention demeure faible, chaque type d'élément - minéral ou organique transporté par l'eau, ou l'eau elle-même, présente sa propre dynamique. La réalisation de bilans amont-aval sur de courtes périodes de temps - à l'échelle de la journée par exemple - permet de mieux comprendre les interactions entre ces éléments.

\section{TRAVAUX CITES}

BORDES J.-M., LUCCHETA J.-C. et ROCHARD M., 1973. Etude d'un écosystème d'eau courante : le Lot. Thèse 3 e cycle. Toulouse. $152 \mathrm{p}$.

BRANSKI J., 1974. Transport of suspended solids along the Vistula River. J. Nordic Hydrol. 5 (3). 183-192.

DAUTA A., 1975. Etude du phytoplancton du Lot. Thèse 3 cycle. Toulouse. $107 \mathrm{p}$.

GOLTERMAN H.-L., 1973. Deposition of river silts in the Rhine and Meuse delta. Freshwat. Biol. 3 (3). 267-281.

HEINEMANN H.-G., HOLT R.-F. et RAUSCH D.-L., 1973. Sediment and nutrient research on selected corn belt reservoirs in Man-made lakes : their problems and environmental effects. Geographical Monographs 17. 381-386. 
PETROVIC G., TASOVAC T. et RADOSAVLJEVIC R., 1969. Der Schwebstoffgehalt der Donau bei Beograd. Arch. Hydrobiol. (suppl.) 36. 98-108.

PROCHAZKOVA L., 1975. Balances in man-made lakes (Bohemia). Nitrogen and phosphorus budgets : Slapy reservoir. in Ecological Studies 10 : coupling of land and water systems. 65-73.

ROEHL J.-W et HOLEMAN J.-N., 1973. Sediments studies pertaining to small reservoir design in Man-made lakes : their problems and environmental effects. Geographical Monograph 17. 567-571. 